\title{
Next-Generation Optical Fronthaul in the iCirrus Project
}

\author{
Jörg-Peter Elbers ${ }^{1}$, Jim Zou' ${ }^{1}$ Philippos Asimakopoulos ${ }^{2}$, Nathan Gomes ${ }^{2}$, Kai Habel ${ }^{3}$, Volker Jungnickel ${ }^{3}$, \\ Gregor Linne $^{4}$, Christoph Juchems ${ }^{4}$, Philippe Chanclou ${ }^{5}$, Patrik Ritoša ${ }^{6}$, Howard Thomas ${ }^{7}$ \\ ${ }^{1}$ ADVA Optical Networking SE, Fraunhoferstr. $9 a, 82152$ Martinsried, Germany. ${ }^{2}$ University of Kent, Canterbury, Kent, England, CT2 7NZ, UK. \\ ${ }^{3}$ Fraunhofer HHI, Einsteinufer 37, 10587 Berlin, Germany. ${ }^{4}$ IAF GmbH, Berliner Straße 52 J, 38104 Braunschweig, Germany. \\ ${ }^{5}$ Orange Labs, 2 Avenue Pierre Marzin, 22300 Lannion, France. ${ }^{6}$ Telekom Slovenje, Cigaletova ulica 15, 1000 Ljubljana, Slovenia. \\ ${ }^{7}$ Viavi Solutions, London Road, Newbury Berkshire, RG14 2PZ, UK. \\ jelbers@advaoptical.com
}

\begin{abstract}
We discuss next-generation fronthaul solutions for 5G and legacy radio access networks. Architectures, findings and experimental results from recent lab and field trial activities are reported.

OCIS codes: (060.2330) Fiber optics communications; (060.4250) Networks
\end{abstract}

\section{Introduction}

The $5^{\text {th }}$ generation mobile network $(5 \mathrm{G})$ is much more than just a new radio (NR) interface [1]. Set out to serve diverse markets and industries such as automotive, manufacturing, energy, eHealth, and entertainment, it needs to deliver services with very different bandwidth, latency and reliability characteristics; enhanced Mobile Broad-Band (eMBB), ultra-Reliable Low-Latency Communications (uRLLC), and massive Machine-Type Communications (mMTC) have to be provided over a single network. Fulfillment of 5G performance metrics [2] such as 1000 times higher cell capacity, 100 times higher peak data rates, 10 times lower latency and 10 times better reliability compared to $4 \mathrm{G}^{1}$ requires both technology and architecture innovation.

Network slicing, edge computing and network function virtualization (NFV) will turn the radio access network (RAN) into a programmable resource. A cloud-RAN (C-RAN) architecture boosts efficiency by centralized resource pooling and can be complemented by distributed networking, storage and compute functions as network or application performance dictates. One of the main C-RAN implementation challenges lies in the fronthaul network that connects central units with remote units. In this paper, we investigate a next-generation optical fronthaul based on Ethernet. We discuss architectures and report results of the Horizon2020 iCirrus project.

\section{Architectural considerations}

In today's RAN, optical fronthaul and backhaul are served by different technologies: Backhaul links use standard Ethernet technology. Fronthaul links rely on layer 1 protocols such as CPRI (Common Public Radio Interface) to transport digitized I-Q, control \& management, and synchronization data. Originally designed for connections from the base of a cell tower to the antenna on top, CPRI imposes stringent requirements on bandwidth, latency and admissible time error. A more than 10-fold higher data rate (compared to the user data), a one-way latency of less than $75 \mu \mathrm{s}^{2}$, a timing error of less than $\pm 8 \mathrm{~ns}$, and a frequency error of less than $2 \mathrm{ppb}$ are common specifications for CPRI transport [3]; fulfilling these in a C-RAN context requires dedicated fiber links, posing economic and operational challenges to service providers. Moving from LTE to 5G, it becomes unsustainable to simply follow the CPRI route for C-RAN, as the radio bandwidths would require transport bit-rates in excess of $100 \mathrm{~Gb} / \mathrm{s}$ to each remote unit.

Next-generation fronthaul solutions offer convergence on the basis of a common Ethernet layer [4]. Using different transport profiles, front-, back- and mid-haul (between macro-cell and subtended small cell sites) traffic can be transported over the same (x-haul) network. This approach eliminates stovepipe implementations and allows the use of established Ethernet OAM (operation, administration \& maintenance) tools. By introducing new functional splits [5], the transmission of baseband bits or frequency domain I-Q symbols instead of time domain waveforms leads to a reduction in transport bit-rates compared to CPRI. The fronthaul becomes dependent on the user traffic and statistical multiplexing in the transport network can be exploited while the benefits of central resource pooling and coordination can (at least partly) be retained.

Following current IEEE P1914.1 terminology [6], the fronthaul transport network can be split into a central unit (CU), distributed unit (DU) and remote unit (RU). Depending on deployment scenario, the DU can be combined with either CU or RU. Transport between these units is provided by the next-generation fronthaul interface (NGFI), which exists in two versions.

NGFI-II relates to the higher layer split (HLS) in 3GPP (F1 interface) [7] and describes the fronthaul segment connecting the CU to the DU. The agreed HLS (3GPP Option 2) is derived from the LTE dual connectivity (DC)

\footnotetext{
${ }_{1}^{1} 1$ million devices or $10 \mathrm{~Tb} / \mathrm{s}$ per $\mathrm{km}^{2}, 10 \mathrm{~Gb} / \mathrm{s}$ peak rate, $99.999 \%$ availability, $1 \mathrm{~ms}$ user plane latency.

${ }^{2}$ If support of coordinated multi-point transmission (CoMP) is not necessary, a relaxation to approx. $250 \mu \mathrm{s}$ is possible.
} 
configuration. It separates the radio protocol stack such that the PDCP and higher layer functions are located in the CU, while RLC and lower layer functions are located in the DU. The HLS increases bit-rates by less than $10 \%$ compared to those in the backhaul and has comparable latency and timing requirements. Yet, it allows centralization of CPU-intensive PDCP tasks and central aggregation (e.g. of 5G and LTE traffic).

NGFI-I relates to the lower layer split (LLS) in 3GPP (future F2 interface) and describes the fronthaul segment connecting the DU to the RU. Multiple LLS options are under consideration in both 3GPP and the new eCPRI standard [8]. No agreement has been reached to converge on a single option yet. A split point inside the PHY (3GPP Option 7.2, eCPRI Split IId+Iu) allows the same distributed massive MIMO (multiple input, multiple output) or CoMP operation as CPRI, but pushes the resource management to the RU thereby offering a 5-10 times reduction in data rate. A MAC-PHY split (3GPP Option 6) further reduces the transport bandwidth reduces transport bit-rates to less than twice backhaul rates (depending on the amount of control traffic between DU and RU), but still allows joint transmission and centralized scheduling. The slightly higher split points among the LLS options offer greater statistical multiplexing as bit-rate requirements scale more directly with load, at the expense of complexity in the decoupling of inter-layer dependencies. Latency and timing requirements stay similar to CPRI for all LLS options as they are more determined by the radio technology (carrier aggregation [CA], transmitter [TX] diversity, MIMO, CoMP) than a particular LLS choice.

The iCirrus optical fronthaul architecture is based on a converged Ethernet network which provides 10GbE connections to the RUs and aggregates traffic from multiple antenna sites in linear, chain or ring topologies using $100 \mathrm{GbE}$ trunk links. It can accommodate variable split points based on operator requirements, cell load and aggregation levels (flexible RAN concept). Legacy CPRI signals can be transported over the same network after Ethernet encapsulation (CPRIoEth) by the IEEE P1914.3 Radio-over-Ethernet (RoE) standard [9]. As low latency and timing error is crucial for network performance, frequency syntonisation and time synchronisation is necessary, which can be provided by the IEEE $15882 \mathrm{v} 2$ precision timing protocol (PTP) in conjunction with synchronous Ethernet (SyncE) (using e.g. the ITU-T G.8275.1 telecom profile).

\section{Theoretical investigations}

Based on the architecture described above, work in iCirrus focused on time-sensitive networking approaches meeting the latency and timing error requirements of next-generation x-haul networks. Maximum latency and time error values for the different splits are still under discussion in 3GPP and the eCPRI group. Table 1a summarizes maximum transport latency values used iCirrus [10] and in the most recent eCPRI specification [8], binned into three classes of service (CoS). Current requirements are based on LTE time-division duplex (TDD) data. An eCPRI update taking into account $5 \mathrm{G}$ NR information is expected in 07/2018. The fiber transmission delay $(5 \mu \mathrm{s} / \mathrm{km})$ needs to be taken into account in addition. Tolerable time errors are listed in Table 1b. An additional margin on the eCPRI specifications is required if the slave clock for timing recovery is located in the radio equipment, not at the transport edge. The class $\mathrm{C}$ requirement is an absolute time error which should be fulfilled by all radio transport solutions. Classes A+ to B requirements apply to a local radio cluster in the CPRI or LLS case and denote relative errors.

\begin{tabular}{|l|l|l|}
\hline CoS & Example use case & $\begin{array}{l}\text { One-way latency } \\
\text { (iCirrus / eCPRI) }\end{array}$ \\
\hline High & CPRI, LLS user plane (fast) & $75 \mu \mathrm{s} / 100 \mu \mathrm{s}$ \\
\hline Medium & $\begin{array}{l}\text { CPRI, LLS user plane (slow), } \\
\text { C\&M plane (fast) }\end{array}$ & $440 \mu \mathrm{s} / 1 \mathrm{~ms}$ \\
\hline Low & $\begin{array}{l}\text { HLS \& backhaul user plane, } \\
\text { C\&M plane (slow) }\end{array}$ & $60 \mathrm{~ms} / 100 \mathrm{~ms}$ \\
\hline
\end{tabular}

\begin{tabular}{|l|l|l|}
\hline Class & $\begin{array}{l}\text { Maximum time error } \\
|\mathbf{T E}| \text { (iCirrus / eCPRI) }\end{array}$ & Example use case \\
\hline $\mathrm{A}+$ & $10 \mathrm{~ns} / 20 \mathrm{~ns}$ (relative) & MIMO or TX diversity \\
\hline $\mathrm{A}$ & 30ns / 70ns (relative) & Intra-band contiguous CA \\
\hline $\mathrm{B}$ & $\mathrm{n} / \mathrm{a} / 200 \mathrm{~ns}$ (relative) & $\begin{array}{l}\text { Intra-band non-cont. CA } \\
\text { Inter-band CA }\end{array}$ \\
\hline $\mathrm{C}$ & $1360 \mathrm{~ns} / 1100 \mathrm{~ns}$ (absolute) & 3GPP LTE TDD \\
\hline
\end{tabular}

Tab. 1: a) Fronthaul latency requirements, b) maximum permissible timing error.

Mechanisms for time-sensitive Ethernet transport investigated in iCirrus were: Strict priority (SP) queuing (IEEE 802.3br) without and with frame preemption (IEEE P802.1Qbu) as also considered in IEEE P802.1CM (profiles A and B) [11], time-aware shaping (TAS, IEEE 802.1Qbv) and a deterministic latency approach called FUSION [12]. Compared to round-robin scheduling, SP queuing can reduce average frame delay variation, but cannot reduce peak delay variation or worst case latency which are the relevant parameters here [4]. Frame preemption (FP) can reduce the peak delay variation, as it effectively lowers the maximum transmission unit (MTU) of the lower priority traffic. SP with FP may be able to meet class $C$ specifications, but will require additional means such as a play-out buffer with high precision timing to meet the more stringent classes A+ to B requirements [13]. Time-aware shaping can in principle eliminate any packet delay variation of high priority traffic [14] and therefore offers deterministic latency, but requires a very accurate, network-wide flow scheduling. FUSION maintains deterministic latency for high priority traffic by preserving the timing of its frames and filling low priority traffic into the inter-frame gaps thereby 
eliminating the need for any central scheduling. Both, TAS and FUSION incur additional fixed latency, but this could be offset by smaller playout buffers in the radio equipment.

\section{Experimental results}

To verify the theoretical findings, different experimental building blocks were implemented and successfully tested in iCirrus [10]. A common basis for the hardware demonstrators was a flexible FPGA prototyping platform developed in the project (see Fig. 1a). The list summarizes the main experimental results:

- A CPRIoEth mapper was developed to transport CPRI streams over a converged Ethernet network (3GPP Option 8). Measurement results verified a back-to-back latency of $35 \mu \mathrm{s}$ at $2.5 \mathrm{G}$ and $10 \mu$ s at $10 \mathrm{G}$ operation.

- A full LTE virtual RAN (vRAN) DU/RU setup with MAC-PHY LLS (3GPP Option 6) was developed and achieved $266 \mu$ s round-trip delay at $0.4 \mathrm{~Gb} / \mathrm{s}$ fronthaul data rate. The software packet generation is a significant contributor to latency.

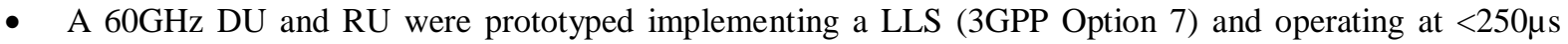
round-trip latency with $1 \mathrm{~Gb} / \mathrm{s}$ throughput. A less bursty packet generation profile would reduce the larger latency values.

- A full LTE vRAN using a HLS (3GPP Option 2) was tested. No impact on the throughput was demonstrated up to $20 \mathrm{~ms}$ Ethernet link latency.

- A deterministic Ethernet aggregator using the FUSION approach was implemented. Measured maximum one-way latency was $14.3 \mu \mathrm{s}$ at $16 \mathrm{kB}$ MTU size and the delay variation was less than 136ns at 10G.

In a final field trial set-up (Fig. 1b) the individual demonstrators were integrated at Telekom Slovenije (TS) facilities. CPRIoEth traffic, $10 \mathrm{GbE}$ best effort traffic, $60 \mathrm{GHz}$ radio, vRAN LTE signals and Femtocell traffic out of the TS core network were aggregated in the central office (CO) and successfully transmitted together with multicasted IEEE 1588 PTP traffic over Telekom Slovenje's metro network to the remote nodes (RN). An IEEE 1588 PTP slave signal was used to generate a $10 \mathrm{MHz}$ synchronization signal for the CPRIoEth demapper. Apart from the additional fiber latency, no additional performance degradations could be observed.
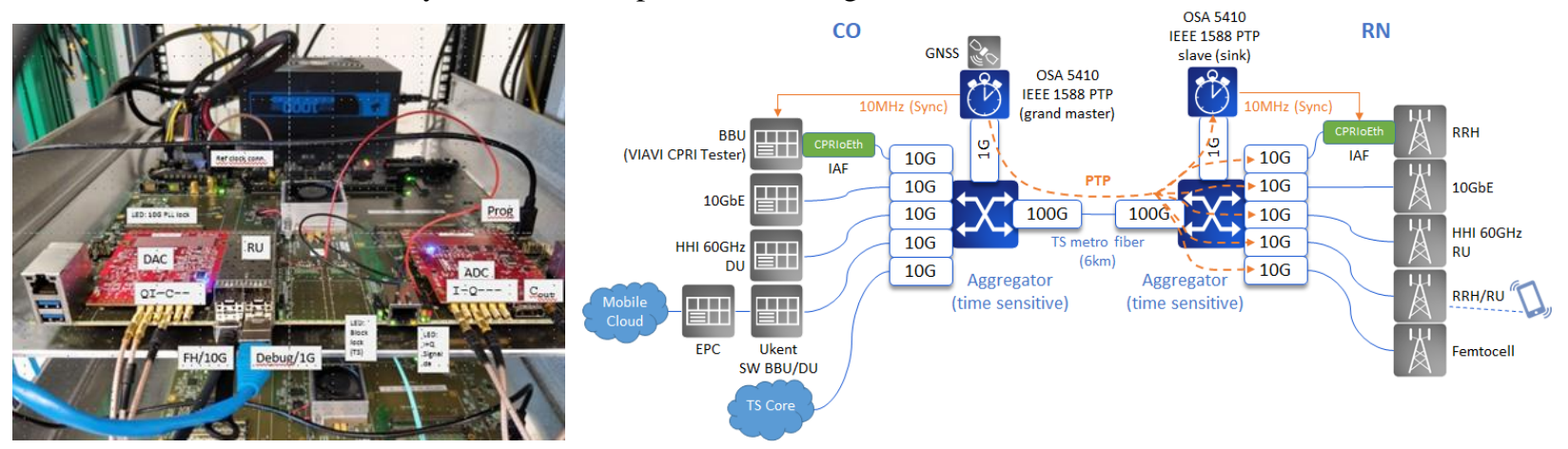

Fig. 1: a) iCirrus 5G FPGA prototyping platform in a $60 \mathrm{GHz}$ RU implementation, b) iCirrus field trial set-up demonstrating multiple radio and split approaches over a converged time-sensitive Ethernet link (right).

\section{Conclusions}

Next-generation Ethernet-based fronthaul networks offer convergence of fronthaul, backhaul and midhaul traffic. We discussed and investigated key building blocks to meet the stringent capacity, latency and timing requirements.

\section{Acknowledgments}

The work leading to these results has received funding from the European Union's Horizon 2020 Research and Innovation Program under Grant Agreement $n^{\circ} 644526$ (iCIRRUS). The authors want to thank Raimena Veisllari, Jan Petter Braute, Steinar Bjørnstad and Mickaël Fontaine from Transpacket for their support.

[1] 5G Infrastructure Association, https://5g-ppp.eu/wp-content/uploads/2018/01/5G-PPP-5G-Architecture-White-Paper-Jan-2018-v2.0.pdf

[2] $5 \mathrm{G}$ Infrastructure Association, http://5g-ppp.eu/wp-content/uploads/2015/02/5G-Vision-Brochure-v1.pdf

[3] Nokia, https://onestore.nokia.com/asset/192728/Nokia_Evolution_to_Centralized_RAN_with_Mobile_Fronthaul_White_Paper_EN.pdf

[4] N. J. Gomes, et al., "Boosting 5G through Ethernet", IEEE Vehicular Technology Magazine, doi: 10.1109/MVT.2017.2782358

[5] 3GPP, TR38.801, "Study on new radio access technologies: Radio access architecture and interfaces", v14.0.0

[6] IEEE P1914.1, "Standard for Packet-based Fronthaul Transport Networks", http://sites.ieee.org/sagroups-1914/p1914-1/

[7] 3GPP, TS 38.470, "NG-RAN; F1 general aspects and principles", v15.0.0

[8] CPRI group, "eCPRI Specification V1.0" and "Requirements for the eCPRI Transport Network V1.1", http://www.cpri.info/

[9] IEEE P1914.3, "Standard for Radio Over Ethernet Encapsulations and Mappings", http://sites.ieee.org/sagroups-1914/p1914-3/

[10] iCirrus, Deliverable D5.4, "Validation test results and analysis evaluation", http://www.icirrus-5gnet.eu/category/deliverables/

[11] IEEE P802.1CM, "Time-Sensitive Networking for Fronthaul”, https://1.ieee802.org/tsn $/ 802-1 \mathrm{~cm} /$

[12] R. Veisllari, et al., "Experimental demonstration of $100 \mathrm{~Gb} / \mathrm{s}$ optical packet network for mobile fronthaul with load-independent ultra-low latency", ECOC 2017

[13] J. Farkas, et al., "P802.1CM simulation results for profiles A \& B", http://ieee802.org/1/files/public/docs2016/cm-farkas-profiles-A-and-B-0316-v01.pdf

[14] M. K. Al-Hares, et al., "Modeling time-aware shaping in an Ethernet fronthaul", IEEE GLOBECOM 2017. 